\title{
A New Approach for Heavy $N$-Doping Process in Ge Epilayers Using Specific Solid Source
}

\author{
Luong Thi Kim Phuong \\ Hong Duc University, Thanh Hoa City, Viet Nam \\ Email: luongthikimphuong@hdu.edu.vn
}

How to cite this paper: Phuong, L.T.K. (2018) A New Approach for Heavy $N$-Doping Process in Ge Epilayers Using Specific Solid Source. Optics and Photonics Journal, 8, 11-19.

https://doi.org/10.4236/opj.2018.82002

Received: January 3, 2018

Accepted: February 8, 2018

Published: February 11, 2018

Copyright $\odot 2018$ by author and Scientific Research Publishing Inc. This work is licensed under the Creative Commons Attribution International License (CC BY 4.0).

http://creativecommons.org/licenses/by/4.0/

(c) (i) Open Access

\begin{abstract}
As an indirect band gap material, Germanium $(\mathrm{Ge})$ has low efficiency of radiative recombination in the wavelength area of about $1550 \mathrm{~nm}$. However, the difference between the direct and indirect band gap is very small $(\sim 140 \mathrm{meV})$ and photoluminescence emission ability of Ge could be greatly enhanced by heavy $\mathrm{n}$-doping process. In this work, Ge growth directly on $\mathrm{Si}(001)$ substrate by molecular beam epitaxial (MBE) technique and a high $n$-doping level in $\mathrm{Ge}$ was obtained owning to using $\mathrm{GaP}$ decomposition source. The $\mathrm{GaP}$ solid source produces $\mathrm{P}_{2}$ molecules instead of $\mathrm{P}_{4}$ molecules, which have higher sticking coefficient than that of $\mathrm{P}_{4}$ comparing to the traditional doping method from $\mathrm{PH}_{3}$ gas precursor molecules. The dependence of optical property on Ge film thickness is also studied. The out-diffusion phenomenon of phosphorus dopant has been observed through SIMS profile analysis. An activated phosphorus concentration can be achieved up to more than $2 \times 10^{19}$ atoms $\cdot \mathrm{cm}^{-3}$ confirmed by Hall effect measurement. This result contributes to realization of Ge-on-Si devices for optoelectronic applications.
\end{abstract}

\section{Keywords}

Germanium, Heavy $N$-Doping, GaP Source, Photoluminescence, Optoelectronic Applications

\section{Introduction}

In the last few years, research on the tensile strained and n-doped Ge thin film on $\mathrm{Si}$ substrate has been the subject of many investigations with the hope to realize an Ge active layer in optoelectronic devices totally compatible with CMOS technology [1] [2] [3] [4] [5]. It has been shown that Ge could become direct band gap material when applying a tensile strained value of $1.9 \%$ on Ge layer [6]. However, Ge will emit a photon with wavelength of about $2500 \mathrm{~nm}$, 
which is out of the wavelength of telecommunication band. Addition, It's a big challenge to get such high tensile strained value while conserving a good quality crystalline of Ge.

Another approach to enhance the efficient radiative recombination of Ge film is fulfilling the energy level of the indirect band gap by doping electron from group $\mathrm{V}$ elements such as $\mathrm{P}$, As or Sb. With a high $\mathrm{n}$-doping level of about $7 \times$ $10^{19}$ atoms $\cdot \mathrm{cm}^{-3}$ [7], the energy state of the indirect band gap, which is equivalent to the lowest energy level at the bottom of the direct band gap is fulfilled. Thus, injected electrons will have a higher probability to occupy the direct $\Gamma$ valley and a high efficiency of radiative recombination would be obtained. Nevertheless, remaining a big challenge is to achieve a high $\mathrm{n}$-doping level due to low solubility of group V-element in Ge [8].

In this study, $\mathrm{P}$ is used for $\mathrm{n}$-doping process because the solubility of phosphorus molecular in $\mathrm{Ge}$ is highest among $\mathrm{V}$-group elements with intermediate temperature range of about $500^{\circ} \mathrm{C}-600^{\circ} \mathrm{C}$ [8]. However, normally method for $\mathrm{n}$-doped Ge film from phosphorus is by mean of using $\mathrm{PH}_{3}$ gas, which produces tetrahedral phosphorus molecule with low sticking coefficient and the highest doping concentration only obtained in the range of $10^{18}-1 \times 10^{19}$ atoms $\cdot \mathrm{cm}^{-3}$. In this work we implement $\mathrm{n}$-doped process by using $\mathrm{GaP}$ specific decomposition solid source, which produce $\mathrm{P}_{2}$ molecule with sticking coefficient 10 times higher than that of $\mathrm{P}_{4}$ [9] [10]. It is shown that activated electron concentration up to 2 $\times 10^{19}$ atoms $\cdot \mathrm{cm}^{-3}$ can be obtained confirming by both Hall effect measurement and band gap narrowing phenomenon.

\section{Experimental Details}

Ge epilayer growth was implemented in a standard MBE system with a base pressure lower than $2 \times 10^{-10}$ torr. The growth chamber is equipped with a 30 $\mathrm{keV}$ reflection high-energy electron diffraction (RHEED) apparatus allowing to observe in-situ and in real-time the Ge growth mode. Ge was evaporated from a two zone heated Knudsen effusion cell with deposited rate in range of about 2 - 5 $\mathrm{nm} \cdot \mathrm{min}^{-1}$.

The substrates were flat, $\mathrm{n}$-type $\mathrm{Si}$ (001) wafers. Cleaning of the substrate surface follows chemical method at the first step with a cycle of oxidation in a hot $\mathrm{HNO}_{3}$ acid and oxide removal in a dilute $\mathrm{HF}$ solution to etch residual carbon contaminants on the surface. After eliminating a rough oxide layer, a very thin and smooth oxide layer is formed in an $\mathrm{HCl}: \mathrm{H}_{2} \mathrm{O}_{2}: \mathrm{H}_{2} \mathrm{O}$ solution to protect the $\mathrm{Si}$ surface from hydrocarbon adsorption during the sample loading process.

The second step is heating process in ultra high vacuum in MBE chamber to evaporate $\mathrm{SiO}_{2}$ thin layer at a temperature of about $650^{\circ} \mathrm{C}$ before flash annealing at $900^{\circ} \mathrm{C}$ in 5 seconds. After this step, the Si surface exposes a well-developed $(2 \times$ 1) reconstruction. The substrate temperature was estimated using a thermalcouple in contact with the backside of the Si wafer with accuracy of about $\pm 20^{\circ} \mathrm{C}$.

The film resistivity was measured at room temperature using a standard four- 
point probe technique. Gold $(\mathrm{Au})$ contacts with a surface of about $3 \mathrm{~mm}^{2}$ were prepared on the top of the Ge surface using conventional optical lithography to insure the reproducibility of the resistivity measurement.

The phosphorus concentration profiles in doped samples were measured by secondary ion mass spectrometry (SIMS), using Cs+ primary ion beams at $1 \mathrm{kV}$ impact energy and with an incidence angle of about $68.4^{\circ}$ from the normal of the sample surface. The phosphorus profiles were calibrated using $\mathrm{P}$ ion implanted Ge samples with various $P$ concentrations; the depth calibration was performed using crater Alpha step depth measurement. The detection limit of our SIMS measurements is below $10^{14}$ atoms $\cdot \mathrm{cm}^{-3}$.

The PL is measured with a $532 \mathrm{~nm}$ laser focused on the sample surface. The PL signal is measured with an InGaAs detector. PL spectra were recorded at room temperature. Active phosphorus concentration is calculated by mean of using Hall effect measurement and reconfirming by band gap narrowing phenomenon.

\section{Results and Discussion}

In order to evaluate the efficiency of $\mathrm{n}$-doping process from the decomposition of $\mathrm{GaP}$, one of the first parameters needing to be controlled is the temperature range of the $\mathrm{GaP}$ cell in which only phosphorus can evaporate. Indeed, $\mathrm{GaP}$ is decomposed into $\mathrm{Ga}$ and $\mathrm{P}_{2}$ molecules and at an intermediate temperature range it is expected that only $\mathrm{P}_{2}$ can escape from the cell while Ga be trapped by a cap placed on top of the cell [10].

Figure 1 displays the evolution of the room-temperature photoluminescence spectrum versus the temperature of the $\mathrm{GaP}$ cell. For all samples, the substrate temperature is chosen to be $300^{\circ} \mathrm{C}$ and the film thickness is $100 \mathrm{~nm}$. The temperature of the $\mathrm{GaP}$ source increases from $600^{\circ} \mathrm{C}$ to $750^{\circ} \mathrm{C}$. After growth, all samples were annealed in the growth chamber at $650^{\circ} \mathrm{C}$ during 60 seconds to activate dopants. As can be seen from the figure, the photoluminescence intensity increases with increasing the temperature of the $\mathrm{GaP}$ source from $600^{\circ} \mathrm{C}$ to $725^{\circ} \mathrm{C}$ and the highest PL intensity is obtained at $725^{\circ} \mathrm{C}$. For the $\mathrm{GaP}$ source temperature at $750^{\circ} \mathrm{C}$, the PL is found to decrease. The decrease of the PL intensity at a temperature higher than $725^{\circ} \mathrm{C}$ can be explained due to the presence of a tiny amount of Ga, escaped from the cell and incorporated in Ge films. The Ga concentration is probably below the detection limit of SIMS apparatus. As Ga acts as a p-type doping in $\mathrm{Ge}, \mathrm{Ga}$ atoms can recombine with electrons induced by $n$-doping, and thus reduce the total electron concentration in Ge films. Thus, the PL result indicates that above $725^{\circ} \mathrm{C}$ the $\mathrm{Ga}$ trap from the $\mathrm{GaP}$ cell becomes less efficient.

To investigate the effect of the doping level versus the substrate temperature as well the role of sticking coefficient of $\mathrm{P}_{2}$ on $\mathrm{Si}$ substrate, we have therefore kept the $\mathrm{GaP}$ source at a constant temperature of $725^{\circ} \mathrm{C}$. Figure 2 displays the evolution of the film resistivity versus the substrate temperature. We note that 


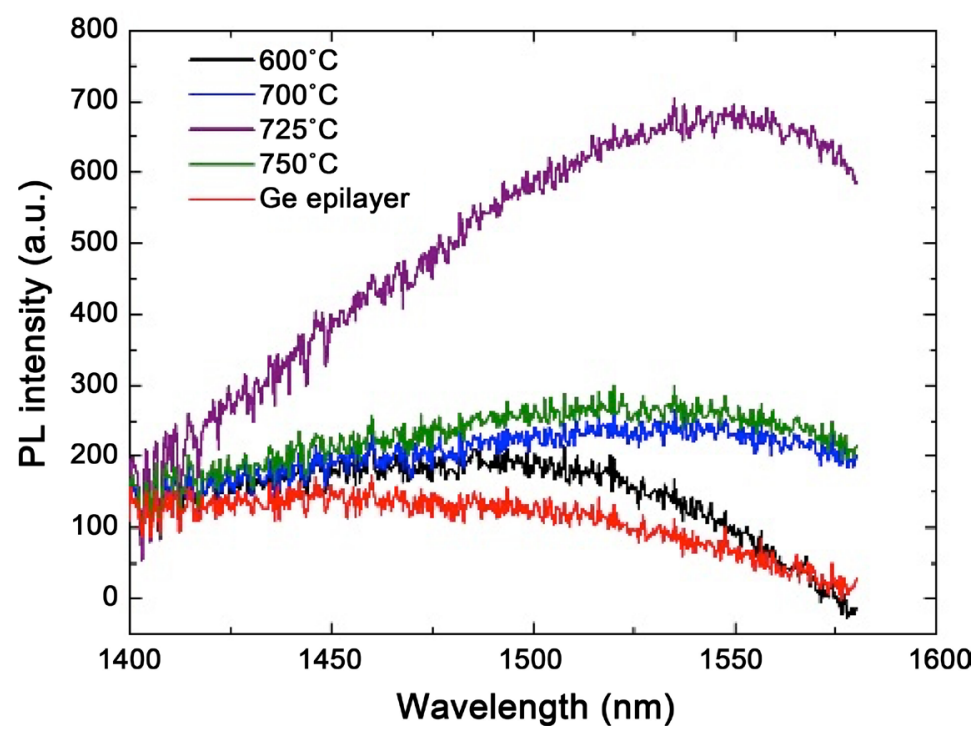

Figure 1. Evolution of the room-temperature photoluminescence spectrum versus the temperature of the GaP cell.

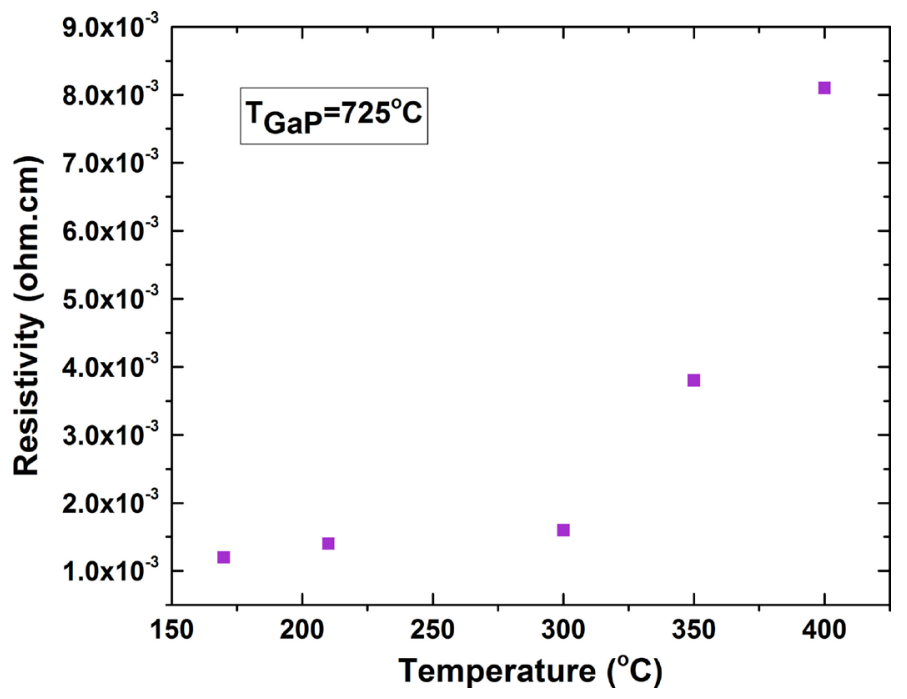

Figure 2. Evolution of the Ge layer resistivity versus the substrate temperature.

$\mathrm{I}-\mathrm{V}$ measurements were carried out at room temperature and all samples have a total thickness of $100 \mathrm{~nm}$ (30 nm thick un-doped Ge layer deposited at $300^{\circ} \mathrm{C}$ to form a smooth and strain relaxed buffer layer, followed by a $70 \mathrm{~nm}$ P-doped Ge film deposited at various substrate temperatures). It can be clearly seen that the resistivity decreases when increasing the substrate temperature from $170^{\circ} \mathrm{C}$ to $450^{\circ} \mathrm{C}$ and the lowest resistivity is obtained at a substrate temperature of $170^{\circ} \mathrm{C}$. This result is somewhat in contrast to the reference [11] which indicates that the highest solubility of $\mathrm{P}$ in $\mathrm{Ge}$ is at $580^{\circ} \mathrm{C}$. It is worth noting that based on these data, we have preformed, at the beginning, numerous doping experiences around $580^{\circ} \mathrm{C}$, the highest solubility temperature of phosphorus. However, the optical and electrical results of the corresponding samples were not as good as expected, we thus decided to investigate the $\mathrm{P}$ doping as function of the substrate 
temperature. We believe the above data of the doping solubility in Ge is only valid for a low concentration range of dopants.

To explain our above results, we suppose that the efficiency of phosphorus doping may depend on two main parameters: the dopant solubility in a matrix and the sticking coefficient of dopants on the film surface. These two parameters are probably competing. The sticking coefficient of an atom or molecule on a substrate surface increases with decreasing the substrate temperature. Since our results reveal that $\mathrm{P}$ doping is more favorable at low substrate temperatures, it appears that the sticking coefficient of the $\mathrm{P}_{2}$ molecules is the dominant parameter determining the phosphorus doping level in Ge film.

We also investigate the effect of film thickness on the optical properties of $\mathrm{P}$ doped Ge layers. Figure 3 shows the evolution of the PL spectrum versus the film thickness. Interestingly, the figure reveals that the PL intensity quickly increases more than 5 times when the film thickness increases from 100 to $530 \mathrm{~nm}$ and then slowly increases for further increase of the film thickness from 530 to $1150 \mathrm{~nm}$ (about 1.2 times). Taking into account the deposition time of about 3.5 4 hours that needs to grow a micrometer thick film by MBE, a film thickness of about $500 \mathrm{~nm}$ appears to be a good compromise for Ge applications in optoelectronics. The rapid increase of the PL intensity when increasing the film thickness from 100 to $530 \mathrm{~nm}$ can be explained by an increase of the tensile strain and in particular by a better crystalline quality of the layer. Indeed, as the $\mathrm{Ge} / \mathrm{Si}$ interface region contains a high density of misfit dislocations, when the film becomes thick enough, the optically probed material is far from these defected regions giving a better optical response. We note that for an excitation laser wavelength of $532 \mathrm{~nm}$ used in this work, the penetration length of photons in Ge is in the order of about $10 \mathrm{~nm}$. Thus, mainly a very thin region of the film surface is sensitive to the PL measurements. Therefore, when the film thickness reaches a

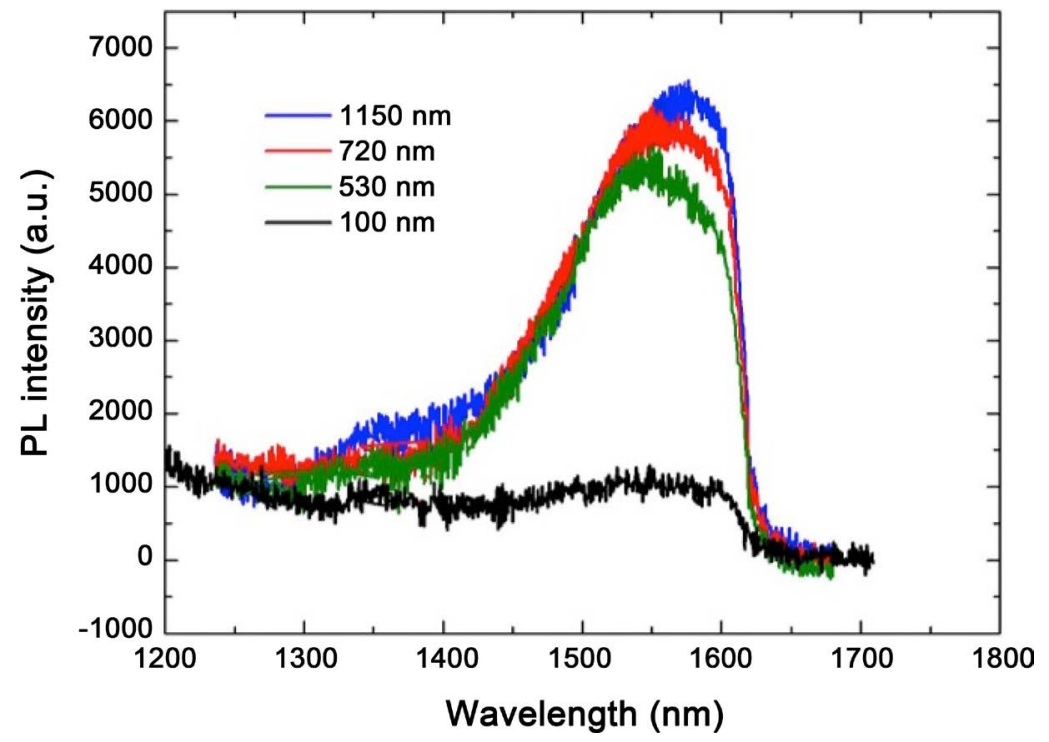

Figure 3. Evolution of the room-temperature photoluminescence spectrum versus the film thickness of P-doped Ge samples. 
value of about $500 \mathrm{~nm}$, the film surface becomes far enough from the interface region and as a consequent, the PL signal will slightly increase with a small increase of the average tensile strain in the film [12].

The above results have allowed us to set up key parameters of the growth condition to obtain efficient emission of the Ge direct band gap, such as the temperature of the doping $\mathrm{GaP}$ source $\left(725^{\circ} \mathrm{C}\right)$, the substrate temperature (around $170^{\circ} \mathrm{C}$ ) and the film thickness (around $500 \mathrm{~nm}$ ). More importantly, the above results imply that the key parameter to get a high electron concentration is the sticking coefficient of the $\mathrm{P}_{2}$ molecule, which is more important than its solubility (which is highest at $580^{\circ} \mathrm{C}$ ).

According to previous studies, when $\mathrm{Ge}$ is under degenerate doping, i.e. when the n-type doping concentration is higher than $1 \times 10^{19}$ atoms $\cdot \mathrm{cm}^{-3}$, a clear red shift in emission wavelength is observed. The phenomenon is called 'band gap narrowing' [13] [14] [15]. Thus, from the shift of the emitted wavelength, one can evaluate the activated electron concentration. In Figure 4 , at $170^{\circ} \mathrm{C}$ of substrate temperature and $725^{\circ} \mathrm{C}$ of $\mathrm{GaP}$ cell, the PL spectrum peak is located at around $1624 \mathrm{~nm}$ (i.e. the corresponding energy is $0.765 \mathrm{eV}$ ). This transition can be attributed to arise from the direct band gap radiative recombination of the $\mathrm{n}$-doped Ge layers. As compared to the energy maximum around $0.810 \mathrm{eV}$ arising from the direct band gap emission of unstrained and un-doped Ge film, we observe here a redshift of $45 \mathrm{meV}$, which can be attributed to band gap narrowing at high $\mathrm{n}$-doping levels. Taken into account a tensile strain of about $0.10 \%$ in our samples (deduced from XR, measurements) and with a maximum of the PL spectrum located at $1624 \mathrm{~nm}$, we can deduce an activated electron concentration of about $2 \times 10^{19} \mathrm{~cm}^{-3}$. The value of the electron concentration is in good agreement with that obtained from Hall measurements shown in Figure 5. We note that for Hall measurements, we have grown thick samples $(1150 \mathrm{~nm})$ on a SOI

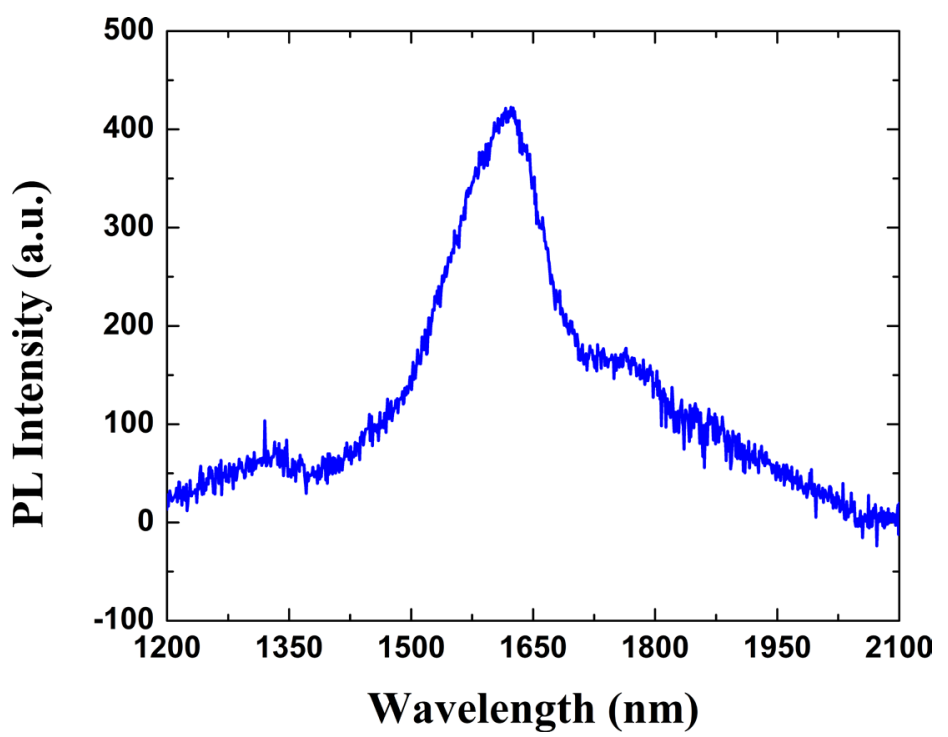

Figure 4. Room-temperature PL spectrum of a Ge layer doped with $\mathrm{P}$. The film thickness is $600 \mathrm{~nm}$, the substrate temperature is $170^{\circ} \mathrm{C}$ and the temperatures of $\mathrm{GaP}$ cell is $725^{\circ} \mathrm{C}$. 


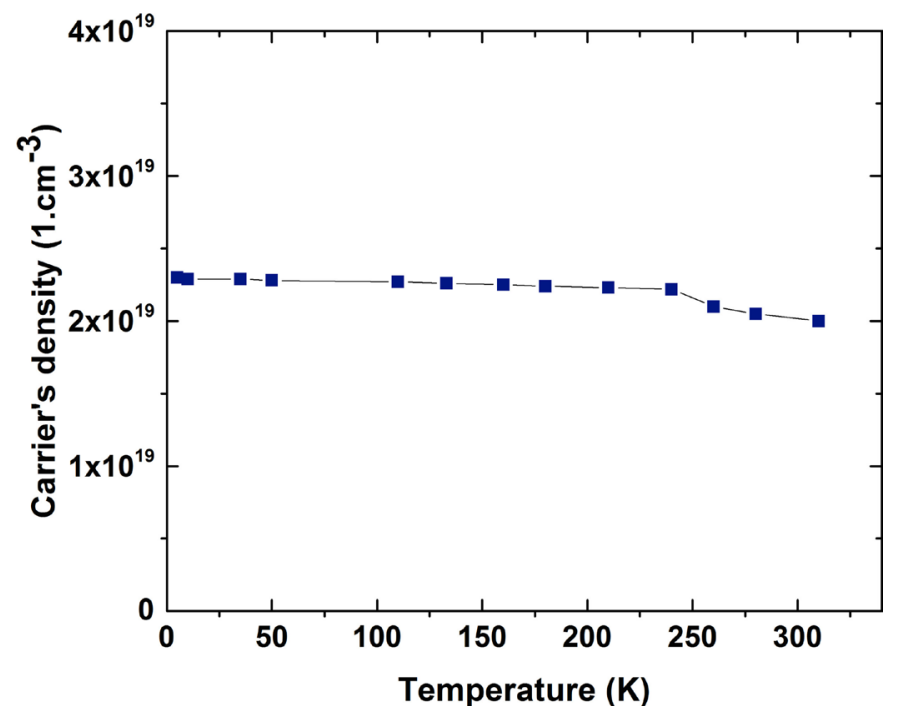

Figure 5. Dependence of carrier's density on measurement temperature.

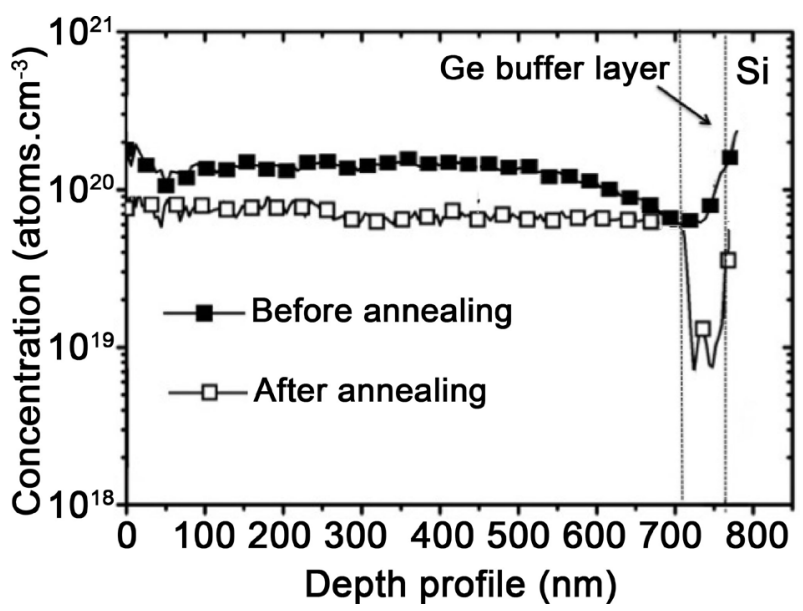

Figure 6. Concentration profiles of $\mathrm{P}$ in Ge epilayers before and after thermal annealing along the film thickness.

substrate (Silicon On Insulator) to avoid any transport contribution coming from the substrate.

For an active diffusion occurring, the temperature should be high enough to overcome energy barrier related to atomic motion. We use rapid thermal annealing at $700^{\circ} \mathrm{C}$ for $60 \mathrm{~s}$ that is proposed in reference [8]. Figure 6 displays SIMS measurements of the $\mathrm{P}$ concentration in Ge film before and after thermal treatment, we can recognize the drop by one order of magnitude of $\mathrm{P}$ concentration compared to the sample before annealing due to the out-diffusion effect. The average concentration of $\mathrm{P}$ is about $7.95 \times 10^{19}$ atoms $\cdot \mathrm{cm}^{3}$ (before annealing, the $\mathrm{P}$ concentration is $1.37 \times 10^{20}$ atoms $\cdot \mathrm{cm}^{-3}$ ) with a little variation along the deposition depth. It is worth noting that according to the Hall measurements, which reveal an activated dopant concentration of about $2 \times 10^{19}$ atoms $\cdot \mathrm{cm}^{-3}$, it means that about $5.95 \times 10^{19}$ atoms $\cdot \mathrm{cm}^{3}$ are still in the interstitial sites that have not been activated yet. 


\section{Conclusion}

A new approach to increase phosphorus doping concentration in Ge has been implemented by using specific GaP solid source. The highest activated electron concentration obtained up to $2 \times 10^{19}$ atoms $\cdot \mathrm{cm}^{3}$ by the Hall effect measurement. This result can be achieved with the following growth condition: the substrate temperature is $170^{\circ} \mathrm{C}$ and the $\mathrm{GaP}$ source temperature is $725^{\circ} \mathrm{C}$. A film thickness of about $500 \mathrm{~nm}$ appears to be a good compromise for Ge applications in optoelectronics. The drop by one order of magnitude of $\mathrm{P}$ concentration occurs before rapid thermal annealing at $700^{\circ} \mathrm{C}$ for $60 \mathrm{~s}$ due to out-diffusion effect.

\section{Acknowledgements}

This research is funded by Vietnam National Foundation for Science and Technology Development (NAFOSTED) under grant number 103.02-2015.106. We also thank Prof. V. Le Thanh and his group at the Aix-Marseille University for supporting this work.

\section{References}

[1] Sun, X., Liu, J.F., Kimerling, L.C. and Michel, J. (2009) Direct Gap Photoluminescence of N-Type Tensile-Strained Ge-on-Si. Applied Physics Letters, 95, 011911011914.

[2] Ishikawa, Y. and Wada, K. (2010) Gemanium for Silicon Photonics. Thin Solid Films, 518, S83.

[3] Liu, J., Camacho-Aguilera, R., Bessette, J.T., Sun, X., Wang, X., Cai, Y., Kimerling, L.C. and Michel, J. (2012) Ge-on-Si Optoelectronics. Thin Solid Films, 520, 3354. https://doi.org/10.1016/j.tsf.2011.10.121

[4] Liu, J., Sun, X., Aguilera, R.C., Kimerling, L.C. and Michel, J. (2010) Ge-on-Si Laser Operating At Room Temperature. Optics Letters, 35, 679.

[5] Luong, T.K.P., et al. (2014) Molecular-Beam Epitaxial Growth of Tensile-Strained and N-Doped Ge/Si(001) Films Using a GaP Decomposition Source. Thin Solid Films, 557, 70. https://doi.org/10.1016/j.tsf.2013.11.027

[6] El Kurdi, M., Fishman, G., Sauvage, S. and Boucaud, P. (2010) Band Structure and Optical Gain of Tensile-Strained Germanium Based on a 30 Band k-p Formalism. Journal of Applied Physics, 107, 013710.

[7] Camacho-Aguilera, R., Cai, Y., Bessette, J.T., Kimerling, L.C. and Michel, J. (2012) High Active Carrier Concentration in N-Type, Thin Film Ge Using Delta-Doping. Optical Materials Express, 2, 146211.

[8] Luong, T.K.P., et al. (2015) Making Germanium, an Indirect Band Gap Semiconductor, Suitable for Light-Emitting Devices. Advances in Natural Sciences: Nanoscience and Nanotechnology, 6, 015013.

[9] Shitara, T. and Ebert, K. (1994) Electronic Properties of InGaP Grown by Solid冈source Molecular Beam Epitaxy With a GaP Decomposition Source. Applied Physics Letters, 65, 356.

[10] Lippert, G., Osten, H.J., Krüger, D., Gaworzewski, P. and Eberl, K. (1995) Heavy Phosphorus Doping in Molecular Beam Epitaxial Grown Silicon with a GaP Decomposition Source. Applied Physics Letters, 66, 3197. 
[11] Madelung, O. (1982) Physics of Group IV Elements and III-V Compounds, Landolt-Börnstein: Numerical Data and Functional Relationships in Science and Technology, Vol 17a, Springer, Berlin.

[12] Luong, T.K.P., et al. (2013) Control of Tensile Strain And Interdiffusion in $\mathrm{Ge} / \mathrm{Si}(001)$ Epilayers Grown By Molecular-Beam Epitaxy. Journal of Applied Physics, 114, 083504.

[13] Camacho-Aguilera, R., Han, Z., Cai, Y., Kimerling, L.C. and Michel, J. (2013) Direct Band Gap Narrowing in Highly Doped Ge. Applied Physics Letters, 102, 152106

[14] Jain, S.C. and Roulston, D.J. (1991) A Simple Expression For Band Gap Narrowing (BGN) in Heavily Doped $\mathrm{Si}, \mathrm{Ge}, \mathrm{GaAs}$ and $\mathrm{Ge}_{\mathrm{x}} \mathrm{Si}_{1-\mathrm{x}}$ Strained Layers. Solid State Electron, 34, 453. https://doi.org/10.1016/0038-1101(91)90149-S

[15] Oehme, M., Gollhofer, M., Widmann, D., Schmid, M., Kaschel, M., Kasper, E. and Schulze, J. (2013) Direct Bandgap Narrowing in Ge LED’s On Si Substrates. Optics Express, 21, 2206. https://doi.org/10.1364/OE.21.002206 